\title{
Impact of self-directed learning readiness and learning attitude on problem-solving ability among Chinese undergraduate nursing students
}

Original article

Ru-Zhen Luo, Xiao-Hong Zhang, Chun-Mei Zhang, Yan-Hui Liu*

School of Nursing, Tianjin University of Traditional Chinese Medicine, Tianjin 301617, China

Received: 15 October 2018; Accepted: 29 December 2018; Published: 20 June 2019

Abstract: Objective: To explore the effects of self-directed learning readiness and learning attitude on problem-solving ability among Chinese undergraduate nursing students.

Methods: A convenience sampling of 460 undergraduate nursing students was surveyed in Tianjin, China. Students who participated in the study completed a questionnaire that included social demographic questionnaire, Self-directed Learning Readiness Scale, Attitude to Learning Scale, and Social Problem-Solving Inventory. Pearson's correlation analysis was performed to test the correlations among problem-solving ability, self-directed learning readiness, and learning attitude. Hierarchical linear regression analyses were performed to explore the mediating role of learning attitude.

Results: The results showed that learning attitude $(r=0.338, P<0.01)$ and self-directed learning readiness $(r=0.493, P<0.01)$ were positively correlated with problem-solving ability. Learning attitude played a partial intermediary role between self-directed learning readiness and problem-solving ability $(F=74.227, P<0.01)$.

Conclusions: It is concluded that nursing educators should pay attention on students' individual differences and take proper actions to inspire students' self-directed learning readiness and learning attitude.

Keywords: undergraduate nursing students $\bullet$ self-directed learning readiness $\bullet$ learning attitude $\bullet$ problem-solving ability $\bullet$ China (c) Shanxi Medical Periodical Press

\section{Introduction}

With the rapid development of medical and health-care services, clinical settings are frequently unpredictable. It is necessary for nurses to improve comprehensive ability as much as possible, especially problem-solving ability. ${ }^{1}$ Problem-solving ability has been viewed as an essential ability for nurses and nursing students in assessing the patient's condition and implementing effective measures. However, a large number of nurses and nursing students lack the ability of problem-solving. ${ }^{2}$ Nevertheless, self-directed learning readiness and learning attitude are the foundation of success for nurses and nursing students, which also influence the problemsolving ability. Therefore, to adopt complex clinical environment and meet the requirement of care, nursing students should improve learning attitude, strengthen learning initiatives, and integrate knowledge and skills. ${ }^{3}$ The purpose of this study was to explore the relationship among self-directed learning readiness and learning

How to cite this article: Luo R-Z, Zhang X-H, Zhang C-M, Liu Y-H. Impact of self-directed learning readiness and learning attitude on problem solving ability among Chinese undergraduate nursing students. Front Nurs. 2019; 2: $x x-x x$

Ә Open Access. @ 2019 Ru-Zhen Luo et al., published by Sciendo. (c) Br-NC-ND This work is licensed under the Creative Commons Attribution NonCommercial-NoDerivatives 4.0 License. 
attitude and problem-solving ability. Thus, nurse educators will adopt relevant strategies to develop students' problem-solving skills so that they can cope up with the challenges they will encounter in their work environment flexibly.

Problem-solving ability refers to an individual's capacity in recognizing problem, making resolutions, and selecting and executing solving strategies. ${ }^{4}$ Problem-solving ability is one of the essential core competencies for nursing students, and it also reflects the nursing students' comprehensive quality. Studies have demonstrated that the problem-solving ability was connected with depression ${ }^{5}$ and suicidal ideation. ${ }^{6}$ Besides, effective problem-solving ability indicated better mental health and higher quality of life. ${ }^{7}$ However, nursing students' ability of solving problems is relatively low; while dealing problems, impulse, carelessness, confusion, and unorganized behaviors would be exposed. ${ }^{8}$ So far, many factors which can influence students' problem-solving ability had been found, but few studies discussed their relationships. Therefore, it is necessary to establish a path model between problem-solving ability and its influence factors among Chinese nursing students.

Taking the initiative to determine needs, develop learning objectives, assess available resources, select appropriate learning methods, and evaluate learning effect is the essential part of self-directed learning. ${ }^{9}$ Selfdirected learning readiness is the extent of learning ability, attitude, and personality characteristics that learners have possessed, ${ }^{10}$ which influences the students' autonomous learning and the choice of teaching methods. ${ }^{11}$ Current studies mainly take self-directed learning and problem-solving ability as assessment criteria to evaluate the effect of new teaching methods such as team-based learning and problem-based learning. ${ }^{2,12,13}$ Moreover, the influencing factors of self-directed learning readiness have been identified, for example, age, grade, those who are enjoying their profession, and so on. In addition, $\mathrm{Li}$ and Yuan reported that there were low correlations between students' self-directed learning readiness and problem-solving ability. ${ }^{14}$ The degree of self-directed learning readiness of nursing student impacts nursing students' autonomous learning, which is also correlated with the core ability of students, such as problem-solving ability. ${ }^{15}$ Although problem-solving ability and self-directed learning readiness are vital for nursing students, few studies concern their relationships.

Learning attitude is a relatively stable psychological tendency in learning situation, which directly affects teaching efficiency. Studies of learning attitude have been widely developed in education, but a few results focused on nursing students. For example, Bernard et al. revealed that nursing students held the negative attitude in caring elderly people, ${ }^{16}$ whereas other researches summarized that personal beliefs, values, culture, personal experiences, and so on can affect the learning attitude of nursing students. ${ }^{17,18}$ Burbank et al. introduced a new teaching strategy to change nursing students learning attitude. ${ }^{19}$ In brief, the present studies focused on the situation of learning attitude, influencing factors, and improving teaching strategies; few researches put forward the impacts of learning attitude on self-directed learning ability and problem-solving ability. Therefore, paying attention to the effects of nursing students' learning attitude on self-directed learning ability and problemsolving ability is imperative.

In summary, problem-solving ability has been received an extensive attention, and the influence of teaching strategies on improving problem-solving ability and the correlations between problem-solving ability and learning attitude or self-directed learning readiness or self-efficacy have been conducted. However, studies did not find the relationships among learning attitude, self-directed learning readiness, and problemsolving ability, and few studies explore the path model of problem-solving ability. If the path model of problemsolving ability can be established among Chinese nursing students, then nursing students will receive the best instruction to improve their problem-solving ability as well as to prepare for nursing career. ${ }^{8}$

Therefore, the purpose of this study was to explore the effects of learning attitude and self-directed learning on problem-solving ability among Chinese nursing students. On the basis of literature reviews, the following five hypotheses were proposed: (1): Chinese undergraduate nursing students were more likely to use dysfunctional dimensions of the problem-solving ability. (2) Learning attitude would have positive influence on the problem-solving ability. (3) Self-directed learning readiness would have positive influence on the problemsolving ability. (4) Learning attitude would have positive influence on the self-directed learning readiness. (5) Learning attitude plays a partial intermediary role between self-directed learning readiness and problemsolving ability.

\section{Materials and methods}

\subsection{Design and sample}

A cross-sectional design was used in this study. The research compared the relationships of self-directed learning readiness, learning attitude, and problemsolving ability among Chinese undergraduate nursing students. The convenience sampling was used. A total of 460 undergraduate nursing students who came from the university in Tianjin, China, were included. Students 
who had finished questionnaires incompletely were excluded from the present analyses. Finally, the analyses were conducted with 429 nursing students, yielding a response rate of $93.26 \%$.

\subsection{Instruments}

\subsubsection{Demographic information of undergraduate nursing students}

Social demographic questionnaire was a self-designed questionnaire, and it was designed to describe a lot of essential information that includes age, gender, grade, and main living location. In this section, the volunteers were also asked whether they had the experience of caring. Besides, their ideal specialty and attitude toward nursing were also involved.

\subsubsection{Self-directed learning readiness}

Self-directed Learning Readiness Scale was developed by Fisher et al.;20 it was translated to Chinese by Wang et al., and its reliability and validity were measured by Wang et al. ${ }^{21}$ In our study, we used the Chinese version of Wang et al. It was used to help evaluate students' self-directed learning attitude and ability. It can also help teachers to evaluate the teaching effect. There are 40 items and three dimensions: self-management (13 items), desire for learning (12 items), and selfcontrol (25 items). Each item was scored on a 5-point Likert scale from 1 (absolutely disagree) to 5 (absolutely agree). The first two dimensions are used to evaluate the ability of self-directed learning, and the desire for learning dimension is used to evaluate the self-directed learning attitude. The total scores range from 40 to 200; higher scores indicate better self-directed learning ability. The Cronbach's a of total scale was 0.849 .

\subsubsection{Attitude to learning}

Attitude to Learning Scale is used to test students' view of learning, which consists of 23 items and four dimensions: learning interest (six items), learning experience (six items), learning habit (seven items), and professional skill (four items). Each item was scored on a 5-point Likert scale from 1 (absolutely disagree) to 5 (absolutely agree). The total scores may range from 23 to 115; higher scores indicate better attitude toward learning. The Cronbach's $\alpha$ of total scale was 0.783 .

\subsubsection{Problem-solving ability}

Social Problem-Solving Inventory was developed by Siu and Shek. ${ }^{4}$ In our study, we used the Chinese version translated by Wang et al. ${ }^{21}$ The scale has 25 items and five dimensions: positive problem orientation (three items), rational problem-solving (seven items), negative problem orientation (five items), impulsivity/carelessness style (five items), and avoidance style (five items). The first two dimensions were used to evaluate the students' problem-solving ability, and the higher score indicates nursing students have high level of problemsolving ability; the other three dysfunctional dimensions were used to evaluate the main defects or shortage existing in the process of problem-solving, and the lower score indicates nursing students have high level of problem-solving ability. The Cronbach's $\alpha$ of total scale was 0.745 .

\subsection{Ethical considerations}

This study was approved by the relevant university ethics committee. The participants were also informed about the purpose of this study. All students voluntarily responded to the questionnaires. Written informed consent was obtained from the participants.

\subsection{Data collection}

The eligible nursing students were invited to participate in the study. The investigators described the purpose of this study and the matters needing attention. The questionnaires were given out face to face. They could return them if they had decided not to participate in the study. Students, who had questions about the study or the questionnaire, were able to contact the investigators. After obtaining the consent of the research objects, the questionnaire was filled out anonymously. All nursing students who took part in this study were asked to complete the questionnaires within 20-30 min. Questionnaires were taken back immediately after completion.

\subsection{Statistical analysis}

The Statistical Package for Social Sciences version 17.0 was used to analyze the statistical data. Descriptive analyses including frequency, percentage, mean, and standard deviation (SD) were performed to analyze demographic variables as well as other variables. Pearson's correlation analysis was performed to test the correlations among dimensions of learning attitude, problem-solving ability, and self-directed learning readiness. Hierarchical regression analysis was used to explore the influence of learning attitude and selfdirected learning readiness on undergraduate nursing students' problem-solving ability. All tests with a $P<0.05$ were considered statistically significant. 


\section{Results}

\subsection{The demographic characteristics of Chinese undergraduate nursing students}

The age of the 429 students ranged from 17 to 25 years, and the mean age was 21.28 years (SD 1.49). Out of 429 participants, $50(11.7 \%)$ were male and 379 $(88.3 \%)$ were female. Almost half of the participants $(48.5 \%)$ mainly lived in villages. Besides, among the participants, 245 (57.1\%) had the experience of caring, $207(48.3 \%)$ took nursing as their ideal profession, and $222(51.5 \%)$ liked nursing specialty. These results are presented in Table 1.

\subsection{The level of self-directed learning readiness, learning attitude, and problem-solving ability among Chinese undergraduate nursing students}

The mean and SD of all study variables are shown in Table 2. The total mean score of self-directed learning readiness was $149.98(S D=15.73)$ and the total mean score of learning attitude was $73.99(S D=11.92)$, which revealed that they were in medium to high levels. The

\begin{tabular}{lr}
\hline General characteristics & $N(\%)$ \\
\hline \hline Gender & $50(11.7 \%)$ \\
Male & $379(88.3 \%)$ \\
Female & \\
Grade & $105(24.5 \%)$ \\
Freshman & $105(24.5 \%)$ \\
Sophomore & $132(30.8 \%)$ \\
Junior & $87(20.3 \%)$ \\
Senior & \\
Have experience of caring & $245(57.1 \%)$ \\
Yes & $184(42.9 \%)$ \\
No & \\
Main living location & $103(24.0 \%)$ \\
City & $118(27.5 \%)$ \\
Town & $208(48.5 \%)$ \\
Village & \\
Ideal profession & $207(48.3 \%)$ \\
Nursing & $222(51.7 \%)$ \\
Non-nursing & \\
Attitude toward nursing specialty & $222(51.5 \%)$ \\
Like better & $185(43.1 \%)$ \\
Neutral & $23(5.4 \%)$ \\
\hline
\end{tabular}

Table 1. Demographic characteristics of nursing students $(N=429)$ item mean score of the positive problem orientation dimension (3.65; $\mathrm{SD}=0.56$ ) was highest, which indicated that students tended to adopt positive measures when encountered problems, and the rational problem-solving dimension was $3.47(\mathrm{SD}=0.57)$. The two dimensions indicated the medium to high levels of problem-solving ability; the item mean scale of negative problem orientation was $3.02(\mathrm{SD}=0.70)$, impulsivity/carelessness style $3.23(S D=0.67)$, and avoidance style $3.59(S D=0.70)$, which reflected that there were quite a number of shortages existing in the process of solving problems.

\subsection{Correlation analysis among self-directed learning readiness, learning attitude, and problem-solving ability}

The results of Pearson's correlation analysis are shown in Table 3. Self-directed learning readiness and learning attitude were significantly and positively correlated with problem-solving ability. Furthermore, all dimensions of the self-directed learning readiness were significantly correlated with all dimensions of problem-solving ability except impulsivity/carelessness style dimension. The professional skill (one dimension of learning attitude) had significant relationships with all dimensions of problem-solving ability.

\subsection{The mediating effect of learning attitude}

Hierarchical regression analyses were used to explore the relationships among self-directed learning readiness,

\begin{tabular}{lccc}
\hline Variables & Mean (SD) & $\begin{array}{c}\text { Item mean } \\
(\text { SD })\end{array}$ & Rank \\
\hline \hline Self-management & $44.79(8.56)$ & $3.45(0.50)$ & 3 \\
Desire for learning & $47.38(5.41)$ & $3.95(0.45)$ & 1 \\
Self-control & $57.81(6.32)$ & $3.85(0.42)$ & 2 \\
Self-directed learning & $149.98(15.73)$ & $3.75(0.39)$ & - \\
readiness & & & \\
Learning interest & $16.73(4.44)$ & $2.79(0.74)$ & 4 \\
Learning experience & $21.91(4.36)$ & $3.65(0.73)$ & 1 \\
Learning habit & $20.76(4.88)$ & $2.97(0.70)$ & 3 \\
Professional skill & $14.59(2.82)$ & $3.65(0.70)$ & 2 \\
Learning attitude & $73.99(11.92)$ & $3.22(0.52)$ & - \\
Positive problem orientation & $18.25(2.79)$ & $3.65(0.56)$ & 1 \\
Rational problem-solving & $17.35(2.83)$ & $3.47(0.57)$ & 3 \\
Negative problem orientation & $15.11(3.48)$ & $3.02(0.70)$ & 5 \\
Impulsivity/carelessness style & $12.92(2.68)$ & $3.23(0.67)$ & 4 \\
Avoidance style & $21.52(4.18)$ & $3.59(0.70)$ & 2 \\
\hline SD standard deviation & & & \\
\hline
\end{tabular}

SD, standard deviation.

Table 2. Comparison of the mean and SDs of variables $(N=429)$ 


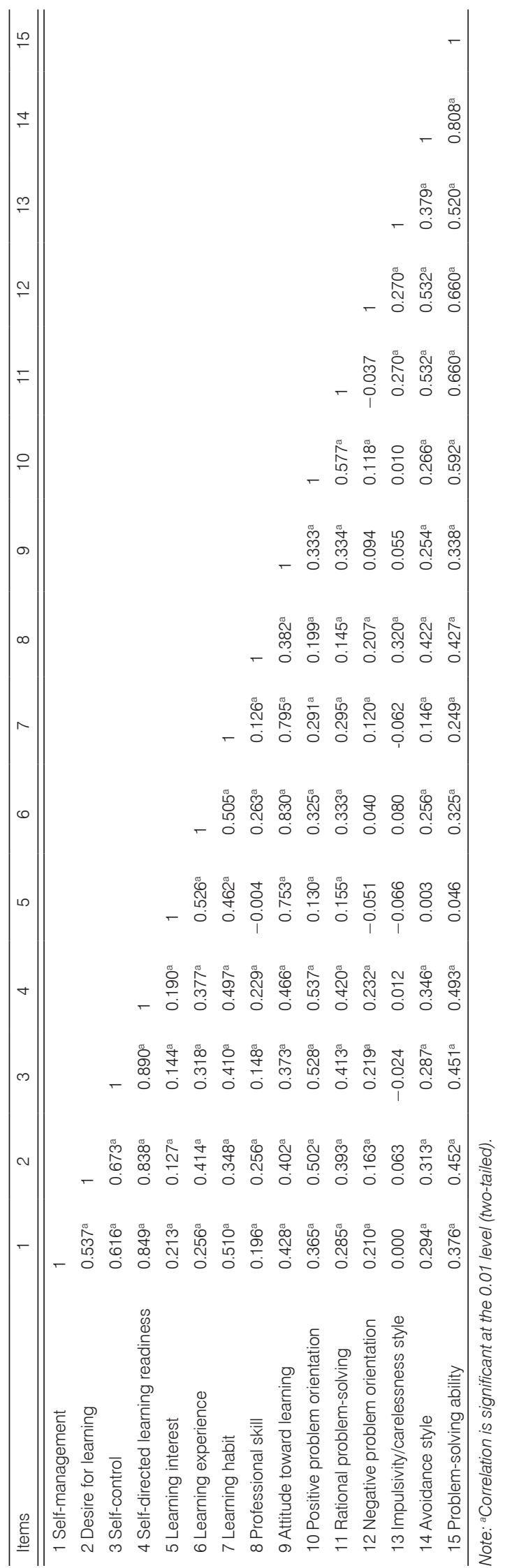

learning attitude, and problem-solving ability. Taking problem-solving ability as the dependent variable, selfdirected learning readiness was entered into the first hierarchical structure of regression equation as an independent variable and learning attitude was placed in the second hierarchical structure, as shown in Table 4. The result showed that self-directed learning readiness could significantly predict problem-solving ability. When learning attitude was added to the equation, $R^{2}$ improved from $24.2 \%$ to $25.5 \%$ and the coefficient of self-directed learning readiness declined from 0.493 to 0.429 , but still reached statistically significant, which indicated that learning attitude played a partial intermediary role between self-directed learning readiness and problem-solving ability (Figure 1$)$. The mediation effect was $(0.466 \times 0.139=0.065, P<0.01)$, direct effect was $(0.429, P<0.01)$, total effect was $(0.493, P<0.01)$, and the mediating effect made up $13.18 \%(0.065 / 0.493)$ of the total effect.

\section{Discussion}

The results of this study reveal that the problem-solving ability of nursing students is at medium to high levels. As shown in Table 2, the score of positive problem orientation is highest in all five dimensions, which explains that the nursing students hold positive attitude toward solving problems. This result is similar to Macao nursing students who were more likely to use the two constructive or adaptive social problem-solving dimensions. ${ }^{22}$ Contrary to our study, Chinese people tend to use the philosophy of doing nothing or using avoidance as a coping strategy when facing problems. ${ }^{4,23}$ In the two constructive or adaptive social problem-solving dimensions, the score of positive problem orientation is higher than rational problem-solving, and it also indicates that nursing students have adequate confidence in handing problems, but they lack rational thought and specific strategy. Hence, to improve problem-solving ability, colleges and educators should focus on cultivating students' positive attitude toward difficulties. Abilities of calm thinking and rational analysis were also taken into account.

The results indicate that the participants have medium to high levels in self-directed learning readiness and learning attitude; similar outcomes have been demonstrated in previous studies. ${ }^{24,25}$ As for selfdirected learning readiness, results are slightly lower than the Australians. ${ }^{13}$ It may relate to the specific education background and cultural background in China. In all dimensions, the score of desire for learning is highest, whereas the self-management is lowest, and Wang also demonstrated the similar results. That is, nursing students are willing to accept new things and challenges. ${ }^{8}$ They also desire to learn independently. 


\begin{tabular}{|c|c|c|c|c|c|c|}
\hline Dependent variable & Independent variable & $B$ & $\beta$ & $t$ & $F$ & $R^{2}$ \\
\hline Problem-solving ability & Self-directed learning readiness & 0.316 & 0.493 & $11.720^{\mathrm{a}}$ & $137.366^{a}$ & 0.242 \\
\hline Attitude toward learning & Self-directed learning readiness & 0.353 & 0.466 & $10.891^{a}$ & $118.616^{a}$ & 0.216 \\
\hline \multirow[t]{2}{*}{ Problem-solving ability } & Self-directed learning readiness & 0.275 & 0.429 & $9.091^{\mathrm{a}}$ & $74.227^{\mathrm{a}}$ & 0.255 \\
\hline & Attitude toward learning & 0.117 & 0.139 & $2.938^{a}$ & - & - \\
\hline
\end{tabular}

Note: ${ }^{a}$ Correlation is significant at the 0.01 level (two-tailed).

Table 4. Hierarchical regression analysis $(N=429)$
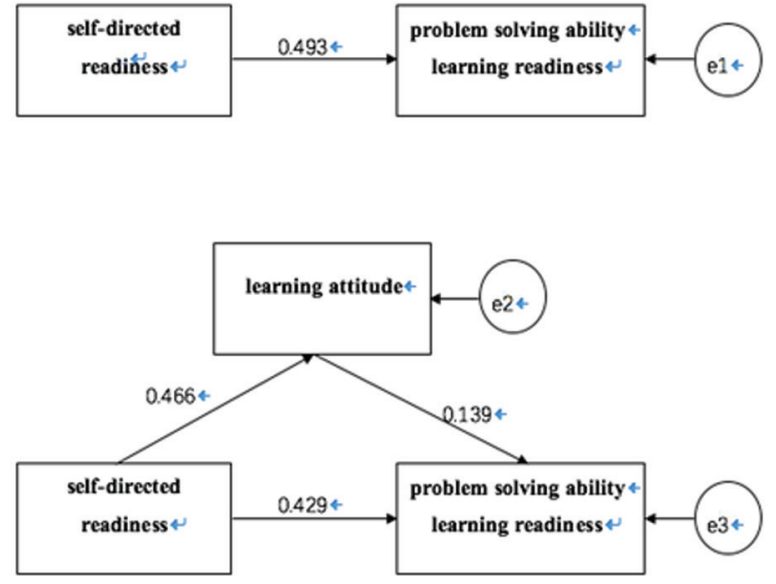

Figure 1. The mediating role of learning attitude in self-directed learning readiness and problem-solving ability.

But their abilities of self-management are poor, such as lacking organization and appropriate way to search useful information. Using appropriate method to carry on the study is also absent. So, it is an effective way to improve self-directed learning readiness by enhancing self-management. Concerning learning attitude, the score of learning experience is the highest, whereas the score of learning habit is the lowest, and Chen also thought that nursing students lack learning interest on the whole. ${ }^{26}$ Implementing openness teaching and cultivating the interest of learning in nursing students can improve their ability of problem-solving.

As shown in Table 4, self-directed learning readiness impacts problem-solving ability. Li and Yuan suggested that the better self-directed learning readiness students are possessing, the better ability of independent learning and problem-solving they will be. ${ }^{14}$ Therefore, it is meaningful for educators to improve students' self-directed learning readiness. As shown in Table 4 and Figure 1, the hierarchical regression analysis further indicates that learning attitude plays a mediating role between the self-directed learning readiness and problem-solving ability. Lee thought that learning attitude positively affects learning ability and teaching effect. ${ }^{27}$ Once the students gained their learning abilities, problem-solving ability will be improved inevitably. Furthermore, there is a strongly positive correlation between learning attitude and self-directed learning ability. ${ }^{25}$ The two studies partly support these results. Nursing educator should focus on students' individual factors and the implementation of personalized teaching. For instance, they can apply new teaching methods such as problem-based learning, team-based learning, and so on to improve students' learning autonomy and attitude. At the same time, showing novel ideas for learning should also be encouraged. Then, nursing students with keen interest will be more likely to solve problems carefully.

The path model of self-directed learning readiness, learning attitude, and problem-solving ability among nursing students has been established, which prompts that nursing educators have different ways to train nursing students' abilities including critical thinking, problem-solving, and self-directed learning. On the one hand, they can improve students' self-directed learning readiness through implementing targeted, flexible, and varied teaching methods. On the other hand, they can accelerate students' satisfaction with profession to improve their learning attitude. In this way, nursing talents who master abundant knowledge and proficient operating skills will be fostered. So, the level of clinical nursing services will be enhanced constantly.

\section{Conclusions}

The results of this study revealed that nursing students have medium to high levels of learning attitude, selfdirected learning readiness, and problem-solving ability. This study provided the evidence linking learning attitude to problem-solving ability among baccalaureate nursing students. The study also proved that learning attitude and self-directed learning readiness were positively correlated with problem-solving ability. The major result was that learning attitude played a partial intermediary role between self-directed learning readiness and problem-solving ability. The results add to our knowledge of personal characters such as learning attitude 
and self-directed learning readiness that can influence nursing students' problem-solving ability. So, nursing educators will improve students' problem-solving ability through changing students' learning attitude and promoting students' self-directed learning ability.

\section{Limitations}

There are some limitations in this study. As a crosssectional design was used, it might not cover all points during students learning in university. Besides, this study used convenience sampling of nursing students only from Tianjin, which may lead to poor representation.

\section{References}

1. Oldenburg NL, Hung WC. Problem solving strategies used by RN-to-BSN students in an online problem-based learning course. J Nurs Educ. 2010;49:219-222.

2. Kim HR, Song $Y$, Lindquist R, Kang HY. Effects of team-based learning on problem-solving, knowledge and clinical performance of Korean nursing students. Nurse Educ Today. 2016; 38: 115-118.

3. Brajtman S, Fothergill-Bourbonnais F, Casey A, Alain D, Fiset $V$. Providing direction for change: assessing Canadian nursing students learning needs. Int J Palliat Nurs. 2007;13:213-221.

4. Siu AM, Shek DT. The Chinese version of the social problem solving inventory: some initial results on reliability and validity. J Clin Psychol. 2005;61:347-360.

5. Prachakul W, Grant JS, Keltner NL. Relationships among functional social support, HIV-related stigma, social problem solving, and depressive symptoms in people living with HIV: a pilot study. J Assoc Nurses AIDS Care. 2007;18:67-76.

6. Becker-Weidman EG, Jacobs RH, Reinecke MA, Silva SG, March JS. Social problem-solving among adolescents treated for depression. Behav Res Ther. 2010;48:11-18.

7. Palermo TM, Law EF, Bromberg M, Fales J, Eccleston C, Wilson AC. Problem-solving skills training for parents of children with chronic pain: a pilot randomized controlled trial. Pain. 2016;157:1213-1223.

8. Wang W. Investigation of nursing students' selfdirected learning ability and problem-solving ability. Fudan University. 2010; 39-43 (in Chinese).

9. Cheng SF, Kuo CL, Lin KC, Lee-Hsieh J. Development and preliminary testing of a selfrating instrument to measure self-directed
Third, self-reported statistical data were used, which may appear to be subjective bias. Therefore, in the future, it is necessary to conduct a longitudinal study and a quantitative research.

\section{Acknowledgment}

We would like to acknowledge all the students who participated in our study.

\section{Conflicts of interest}

All contributing authors declare no conflicts of interest. learning ability of nursing students. Int $J$ Nurs Stud. 2010;47:1152-1158.

10. Wiley K. Effects of a self-directed learning project and preference for structure on self directed learning readiness. Nurs Res. 1983;32:181-185.

11. Chu RJC, Tsai CC. Self-directed learning readiness, Internet self-efficacy and preferences towards constructivist Internet-based learning environments among higher-aged adults. J Computer Assisted Learning. 2009;25: 489-501.

12. Tseng HC, Chou FH, Wang HH, Ko HK, Jian SY, Weng WC. The effectiveness of problem-based learning and concept mapping among Taiwanese registered nursing students. Nurse Educ Today. 2011;31:e41-46.

13. Uys LR, Van Rhyn LL, Gwele NS, Mclnerney P, Tanga T. Problem-solving competency of nursing graduates. J Adv Nurs. 2004;48:500-509.

14. Li Y, Yuan J. Relationship between self-directed learning ability and problem-solving ability of nursing undergraduates. J Nurs. 2015; 22:44-47 (in Chinese).

15. Zheng ZH, Chen JX, Zheng LW, Deng LJ. Study on status quo of self-directed learning readiness in undergraduate nursing students and its influencing factors. Chin Nurs Res. 2016; 30:1945-1949 (in Chinese).

16. Bernard MA, McAuley WJ, Belzer JA, Neal KS. An evaluation of a low-intensity intervention to introduce medical students to healthy older people. $J$ Am Geriatr Soc. 2003;51:419-423.

17. Chen HT, Zhao YZ, Lei FF, Song XH. The learning attitude to experimental lesions and the demands to teachers among male nursing students. J Nurs Admin. 2009; 9:10-12 (in Chinese).

18. Lovell M. Caring for the elderly: changing perceptions and attitudes. J Vasc Nurs. 2006; 24: 22-26. 
19. Burbank PM, Dowling-Castronovo A, Crowther MR, Capezuti EA. Improving knowledge and attitudes toward older adults through innovative educational strategies. J Prof Nurs. 2006;22:91-97.

20. Fisher M, King J, Tague G. Development of a selfdirected learning readiness scale for nursing education. Nurs Educ Today. 2001;21:516-525.

21. Wang W, Cheng Y, Bai JJ. The Chinese version of the self-directed learning readiness among nursing students: some initial results on reliability and validity. Chin J Nurs. 2010; 45: 63-65 (in Chinese).

22. Lau Y. Factors affecting the social problem-solving ability of baccalaureate nursing students. Nurs Educ Today. 2014;34:121-126.

23. Kwok SY, Shek DT. Social problem solving, family functioning, and suicidal ideation among adolescents in Hong Kong. Adolescence. 2009;44:391-406.
24. Yang GF, Jiang XY. The correlation of undergraduate nursing students' self-directed learning readiness and core abilities. Chin J Nurs Educ. 2014; 11:517-519 (in Chinese).

25. Liu YM. Comparative study on English learning autonomy among three grades students' attitude and autonomous learning ability. J Liupanshui Normal Univ. 2016; 28:48-53 (in Chinese).

26. Chen M, Li RL, Wang JJ, Zhang X. Correlation of perceived pressure and general self-efficacy among nursing undergraduate students during early clinical practice. Chin J Nurs Educ. 2017;14:214-217 (in Chinese).

27. Kim HJ, Hong AJ, Song HD. The relationships of family, perceived digital competence and attitude, and learning agility in sustainable student engagement in higher education. Sustainability. 2018;10:4635. 